\title{
Dendritic Spikes and Their Influence on Extracellular Calcium Signaling
}

\author{
MICHAEL C. WIEST, ${ }^{1}$ DAVID M. EAGLEMAN, ${ }^{2}$ RICHARD D. KING, ${ }^{1}$ AND P. READ MONTAGUE ${ }^{1}$ \\ ${ }^{1}$ Division of Neuroscience, Center for Theoretical Neuroscience, Baylor College of Medicine, Houston, Texas 77030; and \\ ${ }^{2}$ Sloan Center for Theoretical Neurobiology, The Salk Institute, La Jolla, California 92037
}

\begin{abstract}
Wiest, Michael C., David M. Eagleman, Richard D. King, and P. Read Montague. Dendritic spikes and their influence on extracellular calcium signaling. J. Neurophysiol. 83: 1329-1337, 2000. Extracellular calcium is critical for many neural functions, including neurotransmission, cell adhesion, and neural plasticity. Experiments have shown that normal neural activity is associated with changes in extracellular calcium, which has motivated recent computational work that employs such fluctuations in an information-bearing role. This possibility suggests that a new style of computing is taking place in the mammalian brain in addition to current 'circuit' models that use only neurons and connections. Previous computational models of rapid external calcium changes used only rough approximations of calcium channel dynamics to compute the expected calcium decrements in the extracellular space. Using realistic calcium channel models, experimentally measured back-propagating action potentials, and a model of the extracellular space, we computed the fluctuations in external calcium that accrue during neural activity. In this realistic setting, we showed that rapid, significant changes in local external calcium can occur when dendrites are invaded by back-propagating spikes, even in the presence of an extracellular calcium buffer. We further showed how different geometric arrangements of calcium channels or dendrites prolong or amplify these fluctuations. Finally, we computed the influence of experimentally measured synaptic input on peridendritic calcium fluctuations. Remarkably, appropriately timed synaptic input can amplify significantly the decrement in external calcium. The model shows that the extracellular space and the calcium channels that access it provide a medium that naturally integrates coincident spike activity from different dendrites that intersect the same tissue volume.
\end{abstract}

\section{N T R O D U C T I O N}

Numerous important neuronal processes are sensitive to external calcium levels, e.g., calcium-dependent cell adhesion (Rose 1998; Tang et al. 1998; Uemura 1998), synaptic plasticity (Bear and Malenka 1994; Denk et al. 1996), neurotransmission (Katz and Miledi 1970; Mintz et al. 1995; Qian et al. 1997), ionotropic receptor function (Xiong et al. 1997), and metabotropic receptor function (Brown et al. 1995; Kubo et al. 1998). Thus if the external calcium level changes significantly during normal neural activity, it could have computational effects by influencing neural function in neighboring regions (Egelman and Montague 1998, 1999; Montague 1996; Smith 1992; Vassilev et al. 1997). In fact, several studies observed significant fluctuations in external calcium at relatively large spatiotemporal scales after elec-

The costs of publication of this article were defrayed in part by the payment of page charges. The article must therefore be hereby marked "advertisement" in accordance with 18 U.S.C. Section 1734 solely to indicate this fact. trical or neurotransmitter stimulation (Benninger et al. 1980; Heinemann et al. 1990; Lucke et al. 1995; Nicholson et al. 1978; Pumain and Heinemann 1985; Stanton and Heinemann 1986) (see Fig. 1). Given that many important computational processes function on millisecond time scales and submicron spatial scales, we were led to ask how electrical events at these smaller scales affect the external calcium level.

In the mammalian brain, action potentials can propagate into the dendrites of cortical and hippocampal neurons (Stuart and Sakmann 1994). These spikes cause large influxes of calcium from the extracellular space (Helmchen et al. 1996; Magee and Johnston 1997; Svoboda et al. 1997). Previous computational work examining the properties of calcium fluctuations around dendrites relied on rough estimates of the magnitude and time course of calcium consumption (Egelman and Montague 1998, 1999). Recent experiments have allowed us to quantify the dynamics of calcium associated with single experimentally measured dendritic spikes in hippocampal and cortical pyramidal neurons (Fisher et al. 1990; Helmchen et al. 1996; Jaffe et al. 1994). These data, which were not incorporated into previous models, allow a more rigorous numerical estimate of how normal dendritic activity at millisecond time scales could influence external calcium.

Using these experimental data and a computer simulation of the extracellular space, we addressed the issue of whether the measured increase in intracellular calcium caused by dendritic action potentials would be paralleled by a significant decrease in extracellular calcium in a region surrounding the activated dendrite. In this study, we examined several influences on external calcium fluctuations during a dendritic spike: 1) channel clustering, 2) background activity, 3) extracellular calcium buffering, 4) the geometric arrangement of coactive dendrites, and 5) dendritic spike modulation by synaptic stimulation.

\section{METHODS}

\section{Strategy}

We approached these issues by using a finite difference model of the extracellular space. The model is shown schematically in Fig. $2 A$. Simulations were constrained by experimental data. We used realistic models of calcium channels derived from patch recordings in hippocampal pyramidal cell dendrites (Fisher et al. 1990; Jaffe et al. 1994), "drove" these models with the voltage waveforms of experimentally measured dendritic spikes (Fig. $2 B$ ), and measured the time course, amplitude, and spatial extent of the external calcium fluctuations that resulted. The segment of dendrite possesses models of 
A

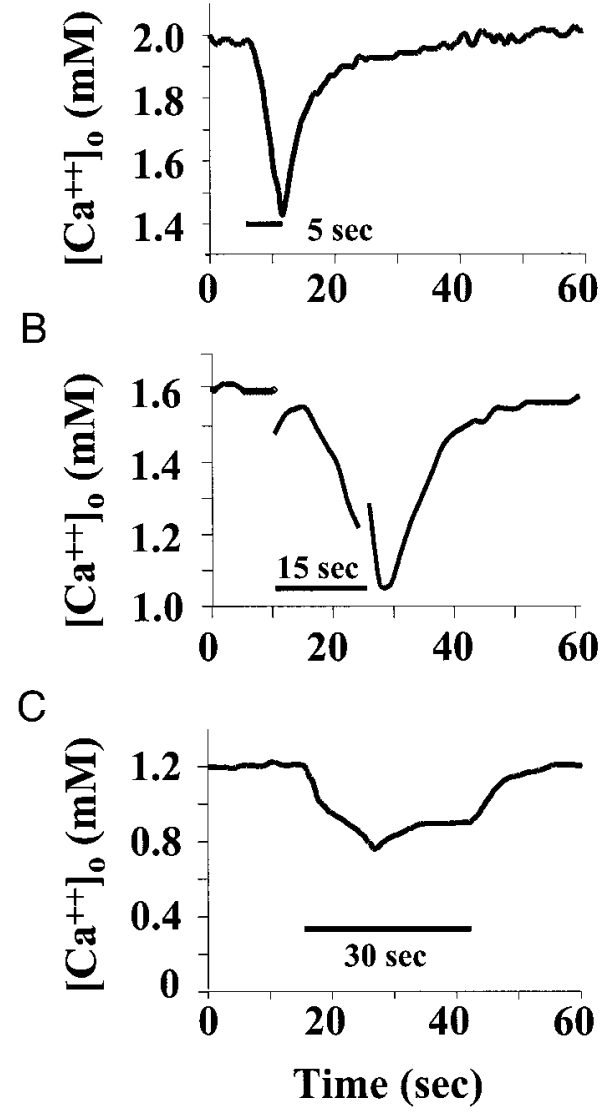

FIG. 1. Neural stimulation decreases external calcium in vivo and in vitro. $A$ : extracellular calcium measured in the stratum pyramidale in a slice of guinea pig hippocampus during $10 \mathrm{~Hz}$ electrical stimulation of the stratum radiatum (black bar). (Adapted from Benninger et al. 1980, Fig. 2, with permission from Elsevier Science.) $B$ : external calcium changes in the stratum pyramidale of area CA1 in rat hippocampus caused by iontophoretic application of $N$-methyl-D-aspartate (black bar). (Adapted from Heinemann et al. 1990, Fig. 3, with permission from Elsevier Science.) $C$ : in vivo recording of external calcium at a depth of $100 \mu \mathrm{m}$ in cat cerebellar cortex during $20 \mathrm{~Hz}$ electrical stimulation (black bar). (Adapted from Nicholson et al. 1978, Fig. 3.)

L-like, N-like, and T-like calcium channels whose operation under a voltage-clamp step is shown in Fig. $2 C$. Figure $2 D$ shows the calcium currents that result when the same dendritic segment is activated with the experimentally measured dendritic spike shown in Fig. $2 B$. The density of the calcium channels was chosen to match the calcium entry per spike to values reported by Helmchen et al. (1996).

\section{Experimentally measured back-propagating spikes}

In our model, the dendritic voltage during a back-propagating spike is clamped to action potential waveforms experimentally measured in a CA1 hippocampal pyramidal cell dendrite $100 \mu \mathrm{m}$ from the cell body (waveforms courtesy of Dax Hoffman). By using this waveform to drive the calcium channel kinetics, we assume that the dendritic calcium currents do not contribute appreciably to the dendritic spike. This assumption is justified by comparing measurements of dendritic action potentials in the presence and absence of calcium channel blockers (Fig. 3; N. Poolos and D. Johnston, personal communication). The difference between the two voltage traces is negligible. We also assumed that an entire segment of dendrite (typically $\sim 4 \mu \mathrm{m}$ in our simulations) is activated synchronously by the back-propagating action potential. The conduction velocity of these dendritic spikes
(>300 $\mu \mathrm{m} / \mathrm{msec}$ ) justifies this assumption (Stuart and Sakmann 1994).

\section{Diffusion in the extracellular space}

To simulate extracellular calcium diffusion, we developed a threedimensional model of neural tissue that includes an explicit representation of the extracellular space (Egelman and Montague 1998, 1999). The basic cubic building blocks, called intracellular units (IUs, 0.826 $\mu \mathrm{m}$ on a side), represent membrane compartments that can be combined to represent dendritic segments (Fig. 2A). In this study, all boundary elements are separated by clefts of $20 \mathrm{~nm}$. The extracellular space is further subdivided into small boxes called extracellular space (ECS) units $(0.118 \mu \mathrm{m}$ on a side). Each ECS unit contains a single state variable that represents the average concentration in that volume. At each time step, an ECS unit updates its concentration as a function of its adjacent ECS neighbors and any calcium consumed or extruded by adjacent IUs. Each IU in the model has set densities of the different calcium channel types, which can be spread evenly over the surface of an IU or clumped arbitrarily to represent the clustering of calcium channels. The total volume of neural tissue simulated was chosen to be large enough to avoid edge effects, typically $(7 \times 0.826 \mu \mathrm{m})^{3}$, where $0.826 \mu \mathrm{m}$ is the side length of an IU.

External calcium diffusion in our model was set to match measurements of the long-range (hundreds of microns) effective diffusion constant in nervous tissue. Because of the impressive variation of those values with brain region, temperature, and experimental method, we show in Fig. $4 A$ the effect of varying our diffusion parameter. We note that the low diffusion constant $\left(8 \mu \mathrm{m}^{2} / \mathrm{s}\right)$ is actually the value measured in rat hippocampus (see Nicholson and Margaret 1987), the particular structure we modeled. This hippocampal value, while apparently atypical, leads to a much larger external calcium decrement during a dendritic action potential.

To determine an effective diffusion constant, it is conventional to define the tortuosity $\lambda$ of a diffusive medium, which relates the effective diffusion constant in the medium to the free diffusion constant in water (or other standard solution):

$$
D_{\text {eff }}=\frac{D_{\text {free }}}{\lambda^{2}}
$$

In our model, the tortousity caused by extracellular geometry is $\lambda=$ 1.23 , as measured by Monte Carlo experiments (Egelman and Montague 1999). Experimenters have found a tortuosity in nervous tissue of $\sim 1.4-1.7$ (Sykova 1997), which means that real tissue slows calcium diffusion more than our model geometry. The difference between our model's geometric tortuosity and the value measured in tissue does not imply any conflict with experiments; rather, it suggests the possibility of slowing factors in the tissue in addition to its winding geometry. In fact, experimental studies have raised the possibility that calcium buffers may act to slow calcium diffusion (Nicholson and Margaret 1987); active calcium channels and physical barriers may also influence the rate of calcium diffusion. To compensate for this difference in tortuosity, we set the local free diffusion constant to $300 \mu \mathrm{m}^{2} / \mathrm{s}$, which is lower than the value measured in solution. This choice yields an effective diffusion constant of 200 $\mu \mathrm{m}^{2} / \mathrm{s}$. This value lies in the middle of the range of effective diffusion constants reported from measurements in tissue (reviewed in Nicholson and Margaret 1987). Further discussion of the model diffusion parameter may be found in Egelman and Montague (1999).

\section{Driving force for calcium entry}

A modified Goldman-Hodgkin-Katz (GHK) model gives the calcium current

$$
I_{\mathrm{Ca}}=\bar{P} \cdot A(V, t) \cdot \frac{4 \mathrm{~F}^{2}}{\mathrm{R} T} \cdot V \cdot \frac{\left[\mathrm{Ca}^{2+}\right]_{\mathrm{o}}-\left[\mathrm{Ca}^{2+}\right]_{\mathrm{i}} \cdot e^{2 \mathrm{FV} / \mathrm{R} T}}{1-e^{2 \mathrm{~F} V / \mathrm{R} T}}
$$


A

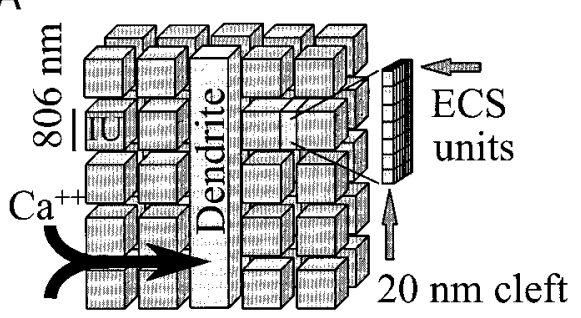

C

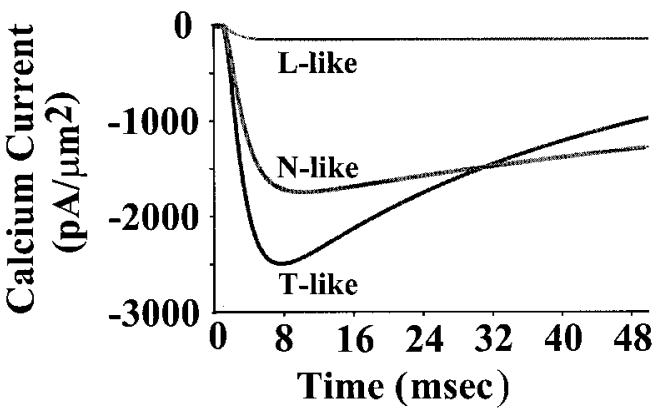

$\mathrm{B}$

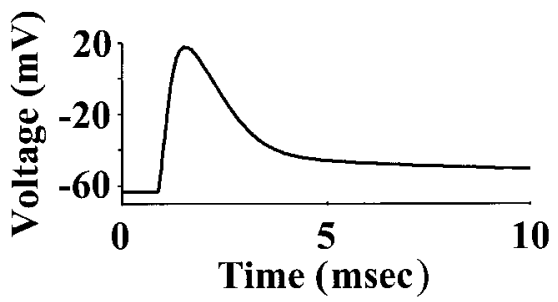

D

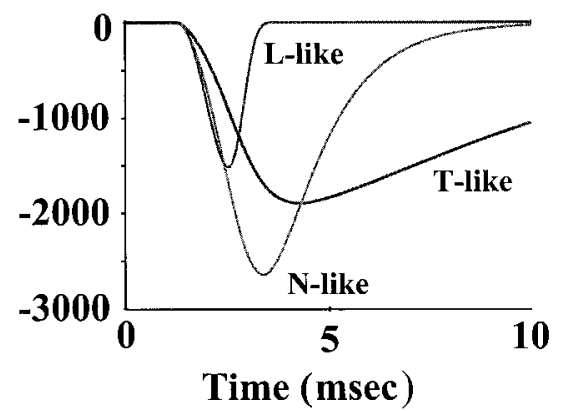

FIG. 2. Computational strategy: extracellular space model and experimental parameters. $A$ : schematic of the finite difference model of the extracellular space. Neural elements such as dendrites are constructed out of cubic intracellular units (IUs), $0.826 \mu \mathrm{m}$ on a side. Calcium channels are placed on the surface of the dendrite and are driven by an experimentally measured voltage trace (B). Extracellular space (ECS) units represent the space between neural elements as clefts $20 \mathrm{~nm}$ thick $(0.118 \mu \mathrm{m}$ on a side). Note the extreme exaggeration of the cleft width. Calcium diffuses through this space with effective diffusion constant $\mathrm{D}=200 \mu \mathrm{m}^{2} / \mathrm{s}$. $B$ : back-propagating action potential used to activate the channel models. This action potential was measured in the apical dendrites of a CA1 hippocampal pyramidal cell $100 \mu \mathrm{m}$ from the soma. $C$ : currents through experimentally fit calcium channel models in response to a voltage clamp step from -80 to $-10 \mathrm{mV}$. Here, the total calcium influx was fixed to 13,600 ions $/ \mu \mathrm{m}^{2}$, as derived from the measurements of Helmchen et al. (1996) (see METHODS). The T-like curve represents the current through low-voltage activated, rapidly inactivating channels. The L-like and N-like curves show the contribution of high-voltage activated, noninactivating, and moderately inactivating channel models. We assume equal densities of the 3 channel types. $D$ : individual model currents when the same channel models are driven by the voltage trace of $B$, with total calcium influx fixed to 13,600 ions $/ \mu \mathrm{m}^{2}$. Under this more physiological stimulus, the more slowly inactivating channels turn off first. The reason is that the high-voltage activated channels (L-like and $\mathrm{N}$-like) are turned off by their activation parameters, which quickly become small when the membrane voltage drops below approximately $-20 \mathrm{mV}$. In contrast, the low-voltage activated (T-like) channels are turned off by their dwindling inactivation parameter because their activation parameter is still sizable on the tail of the dendritic spike $(\sim 0.5$ at $-40 \mathrm{mV})$.

where $\bar{P}$ is the maximum calcium permeability, $V$ is the transmembrane voltage, $\mathrm{F}$ is the Faraday constant, $\mathrm{R}$ is the gas constant, $T$ is the temperature, and $\left[\mathrm{Ca}^{2+}\right]_{\mathrm{i}}$ and $\left[\mathrm{Ca}^{2+}\right]_{\mathrm{o}}$ are the internal and external calcium concentrations. $A(V, t)$ varies between 0 and 1 , representing the channel models in a Hodgkin-Huxley formalism as described in Channel models.

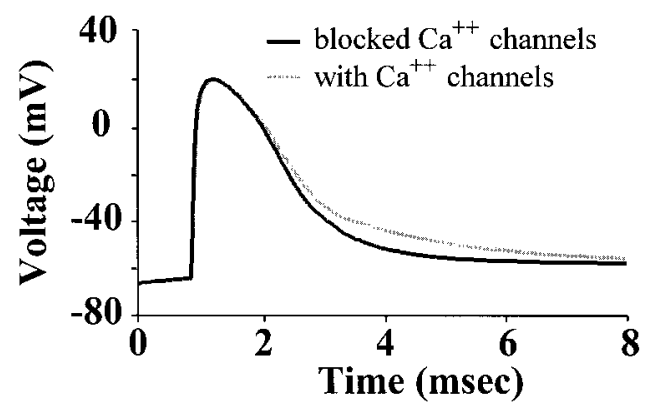

FIG. 3. Justification for neglecting calcium's contribution to dendritic voltage. Recording of a dendritic action potential from a pyramidal cell in rat hippocampal area CA1, with calcium channel blocker (black trace) and active calcium channels (gray trace). The calcium current contributes negligibly to the membrane voltage (adapted from Fig. 2 of Poolos and Johnston 1999).

\section{Channel models}

Although a number of fine distinctions exist among dendritic calcium channels, the three canonical types we used were a transient channel (T-like), a noninactivating channel (L-like), and a moderately inactivating channel (N-like). The Hodgkin-Huxley calcium channel models are represented in a standard way as functions of activation $\left(m_{i}\right)$ and inactivation $\left(h_{i}\right)$ variables that together define a "channel model" $A_{i}(V, t)$ where $i$ indicates T-like, N-like, or L-like calcium channels. The exact form of the channel models and their dependence on experimental data were taken from Fisher et al. (1990) and Jaffe et al. (1994):

$$
A_{T}(V, t)=m_{T}^{2} h_{T} \quad A_{N}(V, t)=m_{N}^{2} h_{N} \quad A_{L}(V, t)=m_{L}^{2}
$$

In all cases, $m_{\infty}(v)=\alpha_{m}(v) /\left[\alpha_{m}(v)+\beta_{m}(\mathrm{v})\right]$, and where applicable $h_{\infty}(v)=\alpha_{h}(v) /\left[\alpha_{h}(v)+\beta_{h}(v)\right]$. The time constants are $\tau_{m}=$ $1 /\left[\alpha_{m}(v)+\beta_{m}(v)\right]$ and $\tau_{h}=1 /\left[\alpha_{h}(v)+\beta_{h}(v)\right]$. The rate parameters are given by $\alpha_{m}(v)=a \cdot(-v+b) /\{\exp [(-v+b) / 10]-1\}, \beta_{m}(v)=$ $c \cdot \exp (-v / d), \alpha_{h}(v)=e \cdot \exp (-v / f)$, and $\beta_{h}(v)=1 /\{\exp [(-v+$ $g) / 10]+1\}$. For the T-like channels, $a=0.2, b=19.26, c=0.009$, $d=22.03, e=1.0 \cdot 10^{-6}, f=16.26$, and $g=29.79$. For the N-like channels, $a=0.19, b=19.88, c=0.046, d=20.73, e=1.6 \cdot 10^{-4}$, $f=48.4$, and $g=39$. For the L-like channels, $a=15.69, b=81.5$, $c=0.29$, and $d=10.86$. 


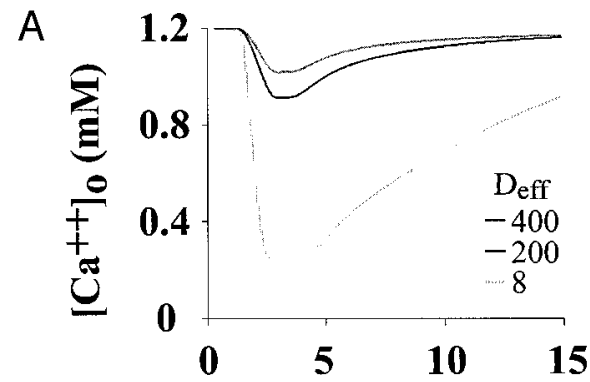

B
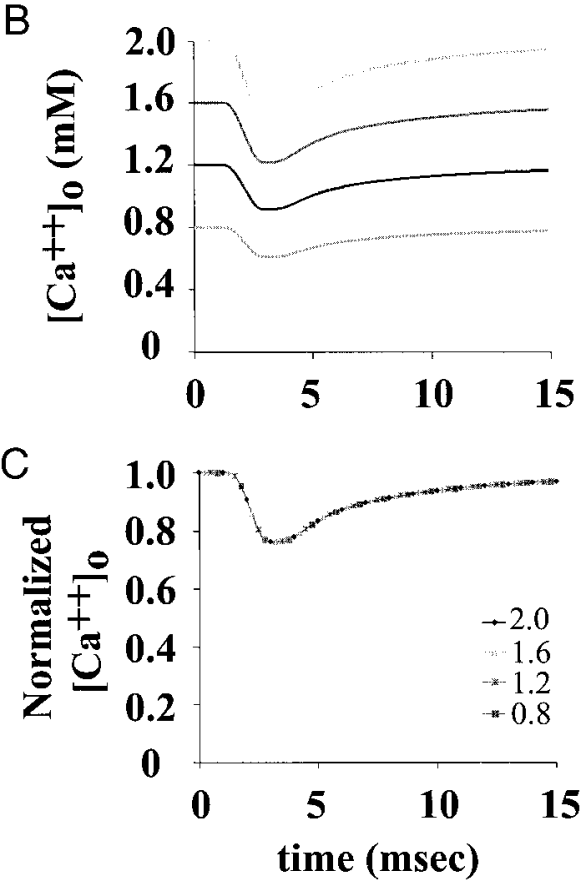

FIG. 4. Effect of varying the diffusion constant and resting external calcium level. External calcium level vs. time calculated by our simulation, activated by the dendritic spike shown in Fig. $2 B$. External calcium concentration is measured in a $20 \times 118 \times 118 \mathrm{~nm}$ volume just outside the center of our model dendritic segment. $A$ : External calcium decrement is shown for three values of the effective calcium diffusion constant: $400 \mu \mathrm{m}^{2} / \mathrm{s}$ (top trace), $200 \mu \mathrm{m}^{2} / \mathrm{s}$ (middle trace), and $8 \mu \mathrm{m}^{2} / \mathrm{s}$ (bottom trace). Note that the slowest diffusion constant $\left(8 \mu \mathrm{m}^{2} / \mathrm{s}\right)$ was the effective value measured in rat hippocampus at $37^{\circ} \mathrm{C}$ (see Nicholson and Margaret 1987), precisely the brain region from which all of our experimental data derived. $B$ : External calcium decrement is shown for 4 values of the initial (resting) calcium level, labeled on the vertical axis. $C$ : Simulation results from $B$ are normalized to the respective initial calcium levels. All results fall on the same curve, demonstrating that the external calcium decrement scales linearly with the initial external calcium concentration in this physiological range. This result is expected, given that we studied transients of a differential equation linear in the external calcium concentration $C$ :

$\frac{\partial C}{\partial t}=\frac{\partial^{2} C}{\partial x^{2}}+($ driving force linear in $C) \cdot G(t)$

$+($ replenishment term linear in $C)$,

where $G(t)$ represents the channel models driven by a spike waveform.

\section{Matching calcium influx to experimental data}

We adjust $\bar{P}$ in the channel models to fix the total calcium consumption through a unit membrane area to agree with the concentration change observed by Helmchen et al. (1996) during a backpropagating spike; if the single-channel maximum permeability is a constant, this is equivalent to adjusting the channel densities. Helmchen et al. (1996) found a $151 \pm 19 \mathrm{nM}$ change in free calcium concentration per dendritic spike and concluded that the observed change represents between $0.5 \%$ and $1.0 \%$ of the total calcium influx, the rest having been buffered. Assuming a dendritic diameter of 1.5 $\mu \mathrm{m}$, these measurements yield a total calcium flux per spike of $6,800-13,600$ atoms $/ \mu \mathrm{m}^{2}$ (depending on whether the $151 \mathrm{nM}$ calcium rise represents $1 \%$ or $0.5 \%$ of the total influx, respectively). We assume equal densities of $\mathrm{N}-$, L-, and T-type channels, with a maximum permeability ratio of $1: 1.38: 1.5$, respectively (Johnston and $\mathrm{Wu}$ 1995). These assumptions yield a total maximum permeability $(\bar{P})$ between 21 and $42 \mu \mathrm{m} / \mathrm{s}$ for a $1 \mu \mathrm{m}^{2}$ patch of membrane.

\section{Resting external calcium level}

To determine the influence of the initial external calcium concentration on the consumption profile, we ran the ECS model with the range of calcium between 0.8 and $2.0 \mathrm{mM}$ (Fig. $4 B$ ). Figure $4 C$ shows that in this physiological range, our results scale linearly with the initial calcium concentration. We used a resting external calcium concentration of $1.2 \mathrm{mM}$ for the remaining simulations, as suggested by Pumain (1998).

\section{Numerical integration}

Our ECS model may be summarized by the difference equation governing the change in the external calcium level, $\Delta C_{j}$, at each time step, $\Delta t$, in each ECS unit $j$. We write this change as a sum of four terms: $X_{j}$ represents diffusion between neighboring ECS volumes, $Y_{j}$ is the calcium consumption into cells through voltage-gated channels, $Z_{j}$ is the replenishment into the ECS by membrane calcium pumps, and $B_{j}$ represents an extracellular calcium buffer

$$
\Delta C_{j}=\left(X_{j}+Y_{j}+Z_{j}+B_{j}\right) \Delta t .
$$

The diffusion term is

$$
X_{j}=\frac{\Delta t}{\delta^{2}} D \cdot \sum_{i=1}^{n}\left(C_{i}-C_{j}\right),
$$

where $D$ is the local diffusion constant, $\delta$ is the distance between the ECS unit centers, and $i$ indexes the $n=12$ neighboring ECS units. The consumption term $Y_{j}$ is proportional to the calcium current given in GHK equation (Eq. I) above. The replenishment term is

$$
Z_{j}=R_{\text {replen }} \cdot N_{j},
$$

where the replenishment rate $R_{\text {replen }}=5 \times 10^{-3} \mathrm{~ms}^{-1}$ and $N_{j}$ is the internal calcium concentration at ECS unit $j$. In simulations that included an extracellular calcium buffer, the free and calcium-occupied buffer concentrations in tissue volume $j$ were labeled $B_{\text {free }}$ and $B_{\text {bound }}$, respectively, and on and off rates are $K_{\mathrm{on}}=5 /(\mathrm{ms} \cdot \mathrm{mM})$ and $K_{\text {off }}=10 / \mathrm{ms}$

$$
B_{j}=K_{\text {off }} \cdot B_{\text {bound }}-K_{\text {on }} \cdot B_{\text {free }} \cdot C_{j} .
$$

The channel models and diffusion equation were integrated numerically using the fourth-order Runge-Kutta method with a time step of $2 \mu \mathrm{s}$. All simulations were programmed in $\mathrm{C}$ and run on Silicon Graphics workstations.

\section{R E S U L T S}

\section{Dendritic spike activity lowers local external calcium}

Figure 5 shows our fundamental result: the change in external calcium through time in response to the dendritic spike shown in Fig. $2 B$. In Fig. $5 A$, the channel models are distributed evenly over the surface of the dendrite. Figure $5 B$ shows that clustering the channels into small patches amplifies the external calcium decrement immediately above a patch. The 
A

Evenly distributed channels
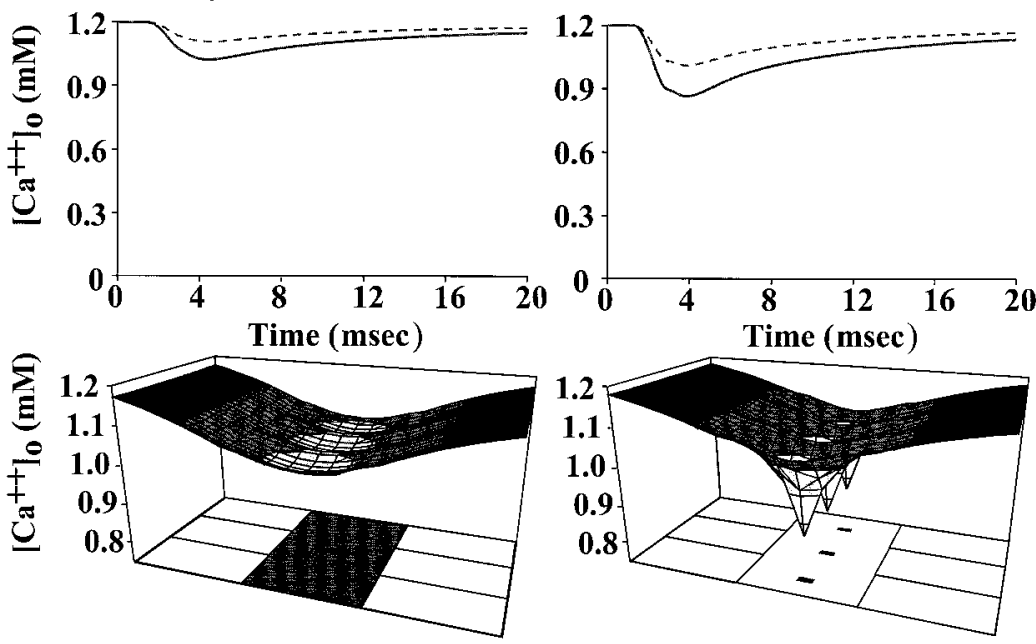

Clustered channels

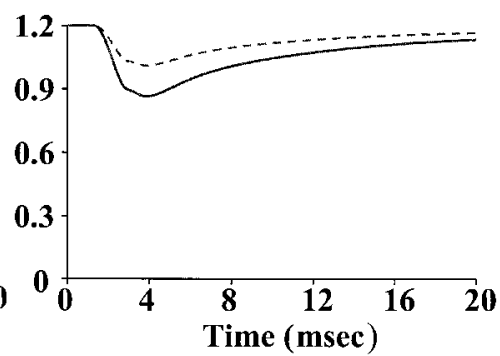

FIG. 5. External calcium fluctuations induced by different arrangements of calcium channels. The dendritic spike used to activate the channel models is shown in Fig. $2 B$. A: external calcium level vs. time calculated by our simulation for uniformly distributed channels. In this example, the calcium flux per spike is bracketed between 6,800 atoms/ $\mu \mathrm{m}^{2}$ (dashed line) and 13,600 atoms $/ \mu \mathrm{m}^{2}$ (solid line). Here, the external calcium concentration is measured in a $20 \times 118 \times 118 \mathrm{~nm}$ volume just outside the center of our model dendritic segment. Bottom: a spatially extended snapshot of the external calcium concentration in a plane parallel to the dendritic surface, measured at $3.94 \mathrm{~ms}$. The orientation of the dendrite is shown as a wide bar on the floor of the graph $(0.826 \mu \mathrm{m}$ wide), the horizontal lines are spaced 0.826 $\mu \mathrm{m}$ apart, and the solid shaded area indicates the location of calcium channels (uniform distribution). Transmembrane calcium flux is 13,600 atoms $/ \mu \mathrm{m}^{2} /$ spike, and we employed equal densities of T-like, N-like, and L-like channels. $B$ : calcium channels are clustered into $118 \times 118 \mathrm{~nm}$ squares, while keeping the average density of calcium channels in the dendritic membrane the same as in $A$. Higher density of channels in the center of the dendrite engenders a larger calcium decrement near the channel cluster. Bottom: same type of snapshot as in A.

bottom panels show that the calcium decrement is localized to within $\sim 1 \mu \mathrm{m}$ of the activated dendrite.

SIGNIFICANCE OF THE CALCIUM FLUCTUATION. The results in Fig. 5 raise a critical question: what magnitude of change in external calcium levels should be deemed significant? To qualify as a signal, a change in extracellular calcium concentration must at least exceed normal statistical fluctuations. If $N$ is the number of ions in the volume of interest, the expected size of the noise is $\sqrt{N}$, assuming the calcium ions act as independent random walkers. Hence, the expected size of a statistical fluctuation in the average external calcium concentration depends on the volume of the averaging region. The results in Fig. 5 show average external calcium concentrations in an extracellular volume $20 \mathrm{~nm} \times 118 \mathrm{~nm} \times 118 \mathrm{~nm}=2.78 \times 10^{5} \mathrm{~nm}^{3}$ (roughly the cleft volume above a typical synaptic active zone). Using the $\sqrt{N}$ measure in a volume of this size, fluctuations $>0.1 \mathrm{mM}$ would be considered significant. Alternatively, if the calcium "reader" were an entire overlying presynaptic terminal, then a reasonable estimate of the averaging volume would be the volume of the entire synaptic cleft, which is about 50 times larger than our present averaging volume (Smith and Augustine 1988). In this case, fluctuations as small as $0.02 \mathrm{mM}$ would be resolvable.

EXTRACELLULAR CALCIUM BUFFERS. It is conceivable that calcium buffers in the extracellular space could change the fluctuation results presented above. To examine this issue, we included a simple buffer in our model of diffusion in the extracellular space (see METHODS). We modeled the extracellular buffer as a first-order binding process with a $K_{d}=2 \mathrm{mM}$, as measured for calcium-binding cadherins (Maurer et al. 1996). Because values for ON and OFF rate constants are not available in the literature, we conservatively chose the on and off rate constants to equilibrate faster than the calcium dynamics. The ON and OFF rates used were $K_{\text {on }}=5 /(\mathrm{ms} \cdot \mathrm{mM})$ and $K_{\text {off }}=10 / \mathrm{ms}$.

It could be predicted that dropping the calcium level in a tissue domain could lead to the release of calcium from buffers, which would reduce the calcium decrement. However, by slowing calcium diffusion from neighboring tissue, buffers might effectively slow the replenishment of a calcium decre- ment, thus amplifying an external calcium decrement. Figure 6 shows that in fact the buffer reduces the amplitude of the calcium decrement, but the signal remains significant even for high buffer concentrations.

BACKGROUND ACTIVITY. We also asked how background neural activity affects the detection of a particular calcium signal. From one point of view, this background activity does not constitute noise in the system because calcium fluctuations caused by "background" activity may be interpreted as signals in their own right. That is, if we consider the external calcium level as a nonspecific measure of local activity, then any activity that affects external calcium is a legitimate signal, as opposed to noise. On the other hand, if we require the system to register the occurrence of a change caused by a specific dendrite in a tissue volume firing at some background rate, the background activity may be interpreted as noise.

Figure 7 shows the calcium level in a tissue volume consisting of a dendrite firing regularly at $30 \mathrm{~Hz}$ surrounded by

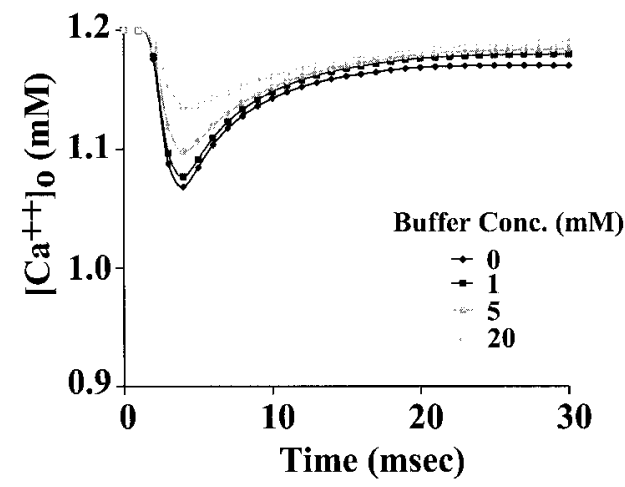

FIG. 6. Simulations of extracellular calcium buffer. Time course of extracellular calcium during a dendritic action potential using 4 concentrations $(\mathrm{mM})$ of calcium buffer in the extracellular space. The membrane permeability was fixed to consume 13,600 atoms $/ \mu \mathrm{m}^{2} /$ spike in the absence of buffer (bottom trace). Buffering is modeled as a first-order process with a dissociation constant of $2 \mathrm{mM}$, corresponding to measurements on the cadherin family of extracellular calcium binding proteins (Maurer et al. 1996). High concentrations of buffer diminish the calcium signal but do not reduce it to insignificance. 


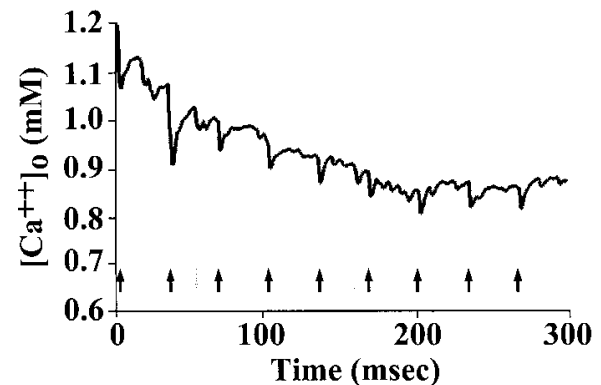

FIG. 7. Effect of neighborhood background activity on the calcium signal at the surface of an active dendrite. The trace shows the calcium concentration outside a simulated dendritic segment firing dendritic action potentials regularly at $30 \mathrm{~Hz}$ (arrows), while the other units (boutons) in the simulated volume fire spikes at an irregular Poisson rate of $10 \mathrm{~Hz}$. The transmembrane calcium flux was fixed to 13,600 atoms $/ \mu \mathrm{m}^{2} /$ spike. Calcium channels were distributed uniformly over the surface of both the dendrite and the boutons. The active volume consisted of a cube containing $125 \mathrm{IUs}$ (each $826 \mathrm{~nm}$ on a side) surrounded by an inactive layer 2 IUs thick. The presence of background fluctuations in external calcium (gray arrows) of comparable size to those caused by the dendrite's activity (black arrows) suggests that the calcium signal is either 1) nonspecific, i.e., fluctuations caused by different cells in a neighborhood are not distinguished, or 2) the calcium signal requires some boosting to be reliably associated with the dendrite.

axon terminals firing independently at Poisson rates of $10 \mathrm{~Hz}$. The calcium level is measured at the surface of the dendrite. Although most of the significant dips in the external calcium concentration are caused by the dendrite's activity, some of the background fluctuations are of comparable size to the dendritic "signal." Thus a calcium sensor just outside the dendrite may not be able to reliably distinguish the dendritic spike signal from other nearby activity. This suggests that if the brain uses extracellular calcium levels to distinguish a signal within a specific diffusion-defined domain (e.g., a signal from a particular dendritic segment), it must boost the signal-to-noise ratio at specific calcium-sensing sites. We summarize some likely mechanisms for boosting the calcium signal in the DISCUSSION. In the absence of such boosting, the calcium signal may be used in a nonspecific, integrative fashion within each diffusiondefined calcium domain. Such domains are expected to be
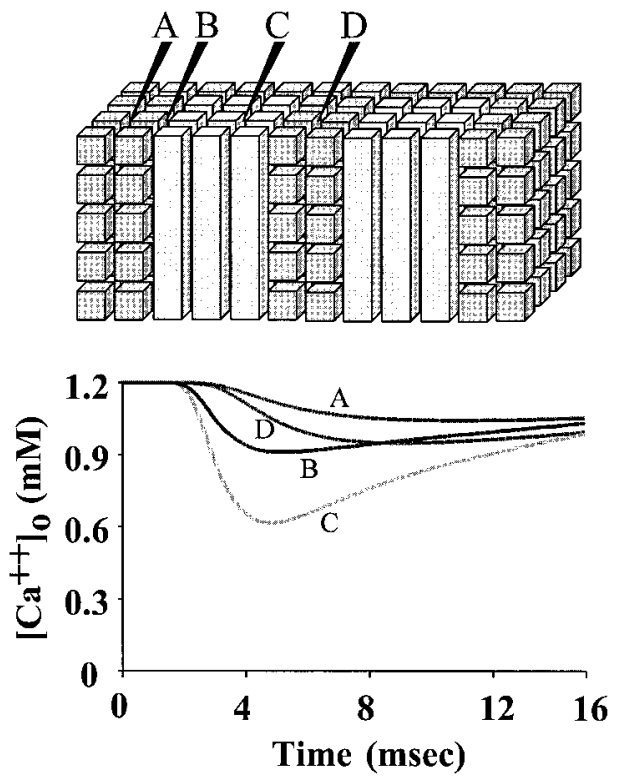

FIG. 9. Coactive dendritic bundles enhance the external calcium decrement. The icon (top) shows the simulated pair of dendritic bundles separated by two $0.826 \mu \mathrm{m}$ units. The plot (bottom) shows extracellular calcium measured at 4 locations (A, B, C, and D) on the picture of the two active bundles. We activated the lighter-colored dendrites with the dendritic spike of Fig. $2 B$, assuming a transmembrane calcium flux of 13,600 calcium atoms $/ \mu \mathrm{m}^{2} /$ spike and equal densities of unclustered T-like, N-like, and L-like channels. In the center of a dendritic bundle (C), there is a very large and sustained calcium decrement. The calcium decrement is larger in the space between two bundles than at the same distance outside of the bundle pair (D vs. A, respectively).

small, given the short range of the calcium fluctuation as shown in Fig. 5.

\section{External calcium may encode synchronous activity from distant cell bodies}

To examine the dependence of the external calcium fluctuation on coincident spike activity of neighboring dendrites, we simulated two representative dendritic arrangements: crossing dendrites (Fig. 8) and dendritic bundles (Fig. 9). Coincident
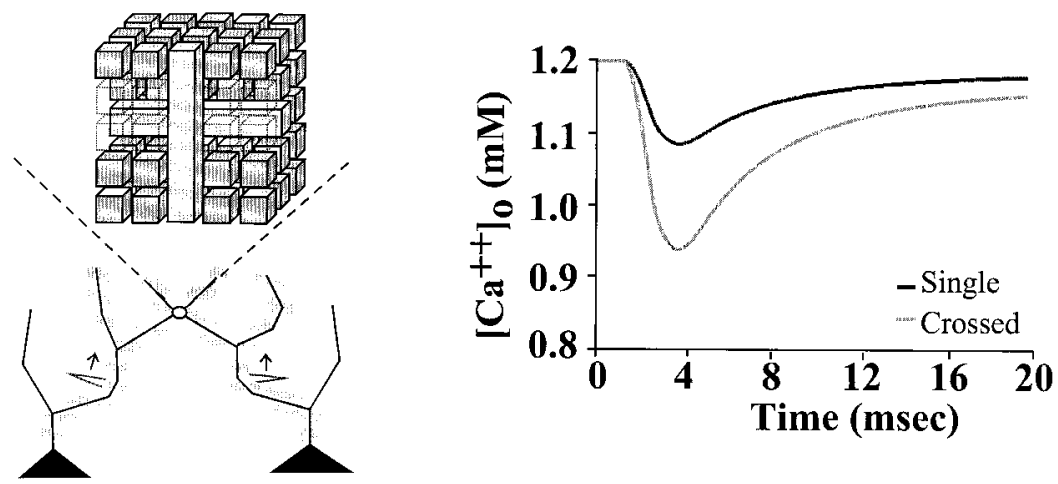

FIG. 8. Intersecting coactive dendrites enhance the external calcium decrement. As mentioned in previous theoretical work, geometric arrangements of dendrites can boost the magnitude of the calcium and time course of the calcium signal (Egelman and Montague 1998, 1999). These effects remain when using realistic channel models. Lower left: geometry of crossing dendrites as might be found in the brain when two dendritic spikes reach an intersection point simultaneously. Upper left: magnified image as simulated by our model; the units in front of the horizontal segment of the crossed pair of dendrites are transparent to display the active segments. Right: external calcium concentration at the center of the intersection region when two crossed dendrites are simultaneously activated by the back-propagating spike shown in Fig. $2 B$ (gray trace). For comparison, the black trace shows the calcium time course resulting from an isolated dendrite (Fig. 4A). Again, we assume a transmembrane calcium flux of 13,600 calcium atoms $/ \mu \mathrm{m}^{2} / \mathrm{spike}$ and equal densities of unclustered T-like, N-like, and L-like channels. 
activity in neighboring dendrites was expected to amplify the external calcium decrement. The degree of the amplification depended on the geometric arrangement of the dendrites. In the case where perpendicular dendrites met at a specific point in space, coincident action potentials in the dendrites amplified the external calcium signal by a factor of two (Fig. 8). Figure 9 shows that bundles of parallel, coactive dendrites (such as those found in Schmolke 1987) can dramatically amplify and prolong the calcium decrement near the center of the bundle at point $C$ (compare to Fig. 5A, solid line), and outside but between a second bundle, point $D$.

\section{Subthreshold synaptic input modulates the external calcium signal}

The amplitude of a back-propagating dendritic spike decreases as it travels away from the cell body. It has been shown that excitatory synaptic input preceding a dendritic spike can boost the spike, save it from attenuation as it travels, and dramatically increase the calcium influx into more distal dendrites (Magee and Johnston 1997). To examine the effect of spike attenuation and boosting in distal hippocampal dendrites, we activated the model with distal $(\sim 250 \mu \mathrm{m})$ dendritic spikes (Fig. 10). The unboosted spike is shown in Fig. 10A (Control spike). Activation of the model with this spike led to a very small external calcium decrement (Fig. 10B) that, by our reasoning above, would not be considered a significant external calcium fluctuation.

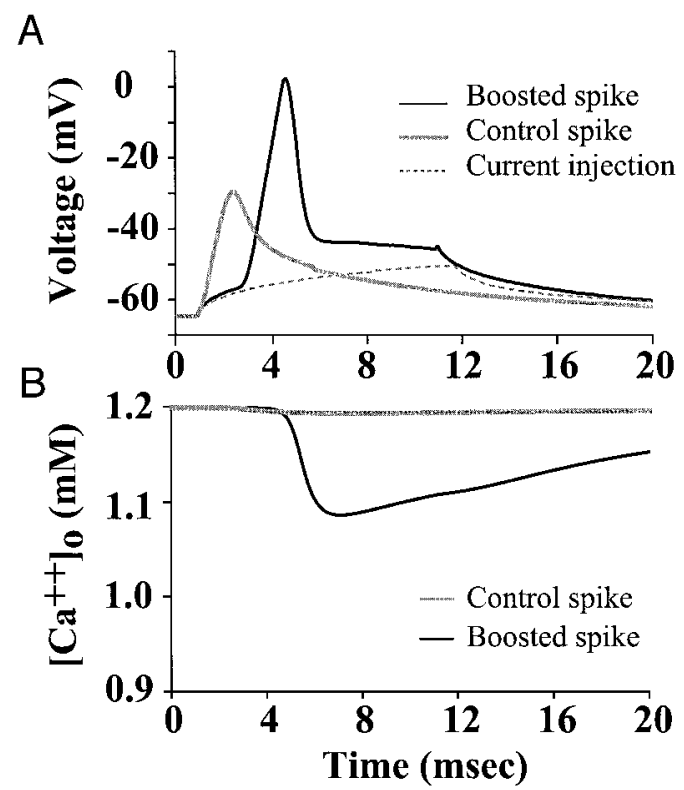

FIG. 10. Dendritic depolarization can boost external calcium signals. A: experimentally measured distal spikes from rat hippocampal CA1 pyramidal cells. Gray trace: distal dendritic spike (measured $\sim 250 \mu \mathrm{m}$ from the soma). Black trace: distal spike paired with dendritic current injection (to simulate synaptic excitation of the dendrite). This pairing boosts the spike amplitude. The same effect occurs for depolarizing synaptic input (see Fig. 10) (Magee and Johnston 1997). Dashed trace: effect of the current injection on membrane voltage in the absence of back-propagating action potentials. $B$ : presence of preconditioning depolarization (synaptic activity or current injection) can also boost the external calcium signal. Time course of external calcium changes for control (gray trace) and boosted (black trace) spikes, assuming a transmembrane calcium flux of 13,600 calcium atoms $/ \mu \mathrm{m}^{2} /$ spike and equal densities of $\mathrm{T}$-like and $\mathrm{N}$-like channels. L-like channels were not included because they tend to be restricted to more proximal dendrites (Magee and Johnston 1997).
A

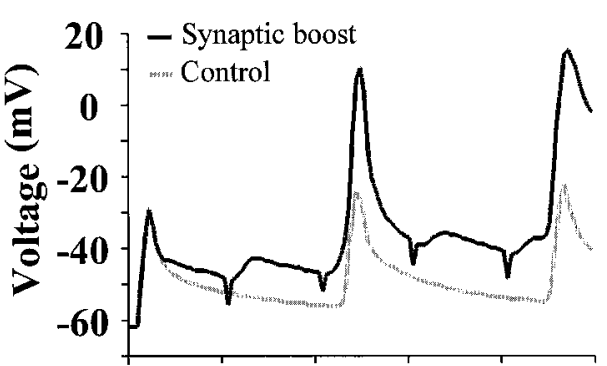

$\mathrm{B}$

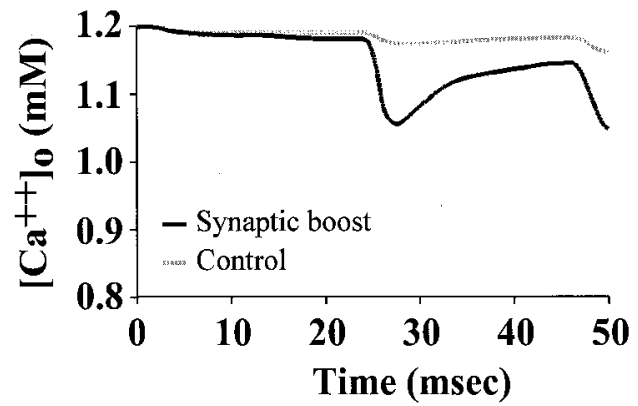

FIG. 11. Excitatory synaptic input also boosts the distal dendritic external calcium signal. A: gray trace, dendrite membrane potential recorded in CA1 hippocampal pyramidal neurons during a spike-generating current injection at the soma (from Fig. $1 B$ of Magee and Johnston 1997). Black trace: synaptic stimulation induces excitatory postsynaptic potentials, which result in supralinear enhancement of the dendritic action potential magnitude when the two coincide. B: gray trace, external calcium concentration outside the simulated dendritic segment activated by the gray trace from $A$. The control voltage trace fails to elicit a significant external calcium fluctuation. Black trace: external calcium concentration associated with the black trace from A. Pairing action potentials with synaptic stimulation in the distal dendrite dramatically enhanced the external calcium signal.

However, as mentioned, distal spikes can be boosted in amplitude by synaptic input or by a depolarizing current in the dendrite that emulates synaptic input (Magee and Johnston 1997; Fig. 10A, boosted spike). Using this boosted dendritic spike to drive the model increased the external calcium decrement (Fig. 10B) to a level comparable to the fluctuations induced by the dendritic spike measured at 100 $\mu \mathrm{m}$ from the soma (Fig. 5). The analogous result caused by experimentally measured synaptic stimulation is shown in Fig. 11, which plots the external calcium level under spikes paired and not paired with distal synaptic stimulation (voltage traces from Fig. $1 B$ of Magee and Johnston 1997). We note that we did not model synaptic dynamics per se; rather we used effective spike waveforms measured during actual synaptic stimulation to drive our model of calcium diffusion in the extracellular space.

This observed boosting effect could be combined with the effect of coincident activity on external calcium to create a mechanism for rapidly indexing specific tissue volumes (Egelman et al. 1998). In the simplest case the mechanism would work as follows. We noted in the previous paragraph that synaptic input to distal dendrites preferentially calls back-propagating spikes into the dendritic branches that received recent synaptic input. Supposing that the intersecting branches in Fig. 8 have just been synaptically excited, each will draw down on external calcium as shown in Fig. 11 , and we might expect that near the intersection of these two branches the calcium decrement would be amplified. 
This expectation is confirmed by the simulation results shown in Fig. 8. Thus synchronous activation of particular synapses could be "flagged" by a deep calcium decrement in a specific tissue volume. This sequence of events provides a mechanism by which to identify a specific pattern of synaptic input impinging on a tissue volume.

\section{I S C U S S I O N}

Using detailed experimental data from the mammalian brain, we have shown that increases in intracellular calcium during back-propagating spikes are likely to be paralleled by concomitant decreases in external calcium near the activated dendrites (Figs. 4-11). The size of such fluctuations can be modulated; for example, we showed that synaptic input could change a small, insignificant external calcium fluctuation into a larger, significant fluctuation. Likewise, clustering of calcium channels amplifies external calcium fluctuations by as much as a factor of two and possibly more (Fig. 5). The most dramatic changes in the external calcium fluctuation are induced by arrangements of neighboring coactive dendrites (Figs. 7 and 8). During a dendritic spike, neighboring, coactive dendrites can amplify the external fluctuation by a factor of four or more. In the same way that the intracellular calcium level can reflect the firing rate of a particular neuron (Helmchen et al. 1996), the extracellular calcium level could encode the amount of coincident spike activity in a population of neurons whose dendrites overlap. Hence, the external calcium changes permit a local comparison of the activities of neurons whose cell bodies may be widely separated and whose activity may be devoted to different computations. Although not investigated in this study, we expect calcium spikes or bursts of action potentials to lead to larger external calcium fluctuations than those caused by a single back-propagating spike.

Although experiments to test directly the predictions of our model require daunting spatiotemporal resolution, experiments with lesser resolution have shown that electrical or neurotransmitter stimulation leads to large, slowly decaying decrements in external calcium (Benninger et al. 1980; Heinemann et al. 1990; Lucke et al. 1995; Nicholson et al. 1978; Pumain and Heinemann 1985; Stanton and Heinemann 1986). These experiments corroborate the hypothesis that neural activity causes significant changes in extracellular calcium at very small spatial and temporal scales, which suggests the need for more definitive experimental tests of rapid external calcium fluctuations.

\section{Physiological readers of external calcium fluctuations}

There are also data to suggest that rapid external calcium signals are functionally important in the moment-to-moment operation of the mammalian nervous system. For example, they may serve to modify synaptic transmission on fast time scales. Experiments in the cerebellum and hippocampus show that changes in probability of release are proportional to the square of external calcium levels (Mintz et al. 1995; Qian et al. 1997). In this way, changing external calcium levels in small tissue volumes could be expressed as relative changes in the probability of release at synaptic terminals within the volume.

Furthermore, metabotropic glutamate receptors in the mammalian brain possess a remarkable structural similarity to the external calcium sensors of the parathyroid gland, suggesting that this important class of glutamate receptor may also act as an external calcium sensor in the brain. Such a possibility has indeed been demonstrated and studied with mutational analysis (Kubo et al. 1998). An ionotropic receptor has also been identified that is activated by changes in external calcium (Xiong et al. 1997).

Another example of a potential reader of external calcium fluctuations that has important neural consequences is the calcium-dependent cell adhesion molecule (cadherin) (see Egelman, D. M., 1998, doctoral dissertation: Computational properties of extracellular calcium dynamics. www.cnl.salk.edu/ eagleman/). These molecules bind to one another in the presence of sufficient calcium and dissociate if calcium levels are low. Mammalian neural tissue expresses several cadherins which are known to be localized to synaptic complexes (Fannon and Colman 1996; Uchida et al. 1996; Yamagata et al. 1995). The role that cadherins play in hippocampal plasticity was tested by Tang et al. (1998) who used peptide antagonists to the binding domain of the cadherins. Application of these peptides to resting hippocampal slices had no effect on baseline synaptic transmission; however, if long-term potentiation (LTP)-inducing stimuli were used, the magnitude of the elicited LTP was dramatically reduced in the presence of the peptides. The peptides had to be present during the induction of LTP; adding the peptides after LTP induction had no effect on the LTP elicited by the stimulation. The interpretation of Tang et al. (1998), which is also our interpretation, is that the stimulation protocol caused decrements in external calcium that allowed the cadherins to dissociate to reveal the sites where the peptides could bind and diminish LTP (induction or expression). The idea is that this inhibitory action arises by preventing reassociation of the cell adhesion molecules. This interpretation predicts that raising external calcium levels might prevent the influence of the peptides on LTP. In fact, raising external calcium from 2.0 to $5.0 \mathrm{mM}$ prevented the inhibitory effects of the peptides.

The activity-dependent fluctuations in the external calcium level predicted by our simulations, as well as experimental measurements of external calcium changes, support the possibility that these external calcium sensors are actually used to signal among cells. Consequently, we suggest that external calcium has a pervasive, fluidlike influence throughout the interstices of the mammalian nervous system, allowing the spaces between neurons and glia to play a fundamental role in the computations carried out in the brain.

We thank Drs. Dan Johnson, Jeff Magee, Dax Hoffman, and Nick Poolos for generously sharing action potential data, calcium channel data, and technical expertise. We also gratefully acknowledge the helpful conversations and technical advice of Drs. David Jaffe and Costa Colbert. Figure 3 was provided by N. Poolos and D. Johnston.

This work was supported by the Center for Theoretical Neuroscience at Baylor College of Medicine, National Institutes of Health (NIH) Grants MH52797 and DA-11723 (P. R. Montague), the United Negro College Fund/ Merck Foundation and NIH grant MH-19547 (R. D. King), and the Biomedical Computation and Visualization Laboratory at Baylor College of Medicine (NSF-BIR-9412521). M. C. Wiest is a Kane Foundation Fellow and is supported by National Library of Medicine Grant 1T15LM07093 through the Keck Center for Computational Biology.

Address for reprint requests: P. R. Montague, Center for Theoretical Neuroscience, Division of Neuroscience, Baylor College of Medicine, One Baylor Plaza, Houston, TX 77030. 
Received 7 July 1999; accepted in final form 20 October 1999. NOTE ADDED IN PROOF

Two recent experimental studies provided a direct demonstration of functional depletion of extracellular calcium in the clefts of individual synapses (Borst and Sakmann 1999; Stanley 2000). Borst and Sakmann (1999) also showed that synaptic calcium depletion leads to changes in synaptic transmission.

\section{REFERENCES}

Bear, M. F. and MalenKa, R. C. Synaptic plasticity: LTP and LTD. Curr. Opin. Neurobiol. 4: 389-399, 1994.

Benninger, C., Kadis, J., And Prince, D. Extracellular calcium and potassium changes in hippocampal slices. Brain Res. 187: 165-182, 1980.

Borst, J.G.G. AND SAKMANN, B. Depletion of calcium in the synaptic cleft of a calyx-type synapse in the rat brainstem. J. Physiol. 521.1: 123-133, 1999.

Brown, E. M., Vassilev, P. M., and Herbert, S. C. Calcium ions as extracellular messengers. Cell 83: 679-682, 1995.

Denk, W., Yuste, R., Svoboda, K., and TanK, D. W. Imaging calcium dynamics in dendritic spines. Curr. Opin. Neurobiol. 6: 372-378, 1996.

Egelman, D. M., King, R. D., AND Montague, P. R. Interaction of nitric oxide and external calcium fluctuations: a possible substrate for rapid information retrieval. Prog. Brain Res. 118: 199-211, 1998.

Egelman, D. M. and Montague, P. R. Computational properties of peridendritic calcium fluctuations. J. Neurosci. 18: 8580-8589, 1998.

Egelman, D. M. and Montague, P. R. Calcium dynamics in the extracellular space of mammalian neural tissue. Biophys. J. 76: 1856-1867, 1999.

FANNON, A. M. AND Colman, D. R. A model for central synaptic junctional complex formation based on the differential adhesive specificities of the cadherins. Neuron 17: 423-434, 1996.

FISHER, R. E., GRAY, R., AND Johnston, D. Properties and distribution of single voltage-gated calcium channels in adult hippocampal neurons. J. Neurophysiol. 64: 91-104, 1990.

Heinemann, U., Stabel, J., and Rausche, G. Activity-dependent ionic changes and neuronal plasticity in rat hippocampus. Prog. Brain Res. 83: $197-214,1990$

Helmchen, F., Imoto, K., AND SAKmann, B. $\mathrm{Ca}^{2+}$ buffering and actionpotential-evoked $\mathrm{Ca}^{2+}$ signalling in dendrites of pyramidal neurons. Biophys. J. 70: 1069-1081, 1996.

JafFe, D. B., Ross, W. N., Lisman, J. E., Lasser-Ross, N., MiyaKawa, H., AND JoHnSTON, D. A model for dendritic $\mathrm{Ca}^{2+}$ accumulation in hippocampal pyramidal neurons based on fluorescence imaging measurements. J. Neurophysiol. 71: 1065-1077, 1994.

Johnston, D. AND Wu, S. M. Foundations of Cellular Neurophysiology. Cambridge, MA: MIT Press, 1995.

KatZ, B. AND Miledi, R. Further study of the role of calcium in synaptic transmission. J. Physiol. (Lond.) 207: 789-801, 1970.

Kubo, Y., MiYashita, T., AND Murata, Y. Structural basis for a $\mathrm{Ca}^{2+}$-sensing function of the metabotropic glutamate receptors. Science 279: 1722-1725, 1998.

Lucke, A., Kohling, R., Straub, H., Moskopp, D., Wassmann, H., and SPECKMANn, E. J. Changes in extracellular calcium concentration induced by application of excitatory amino acids in the human neocortex in vitro. Brain Res. 261: 222-226, 1995.

MAGEE, J. AND Johnston, D. A synaptically controlled, associative signal for hebbian plasticity in hippocampal neurons. Science 275: 209-213, 1997.

Maurer, P., Hohenester, E., AND Engel, J. Extracellular calcium-binding proteins. Curr. Opin. Cell Biol. 8: 609-617, 1996.

Mintz, I., Sabatini, B., AND Regehr, W. Calcium control of transmitter release at a central synapse. Neuron 15: 675-688, 1995.
Montague, P. R. The resource consumption principle: attention and memory in volumes of neural tissue. Proc. Natl. Acad. Sci USA 93: 3619-3623, 1996.

Nicholson, C. and Margaret, R. E. Calcium diffusion in the brain cell microenvironment. Can. J. Physiol. Pharmacol. 65: 1086-1091, 1987.

Nicholson, C., ten Bruggencate, G., Stockle, H., and Steinberg, R. Calcium and potassium changes in extracellular microenvironments of cat cerebellar cortex. J. Neurophysiol. 41: 1026-1039, 1978.

Poolos, N. P. AND Johnston, D. Calcium-activated potassium conductances contribute to action potential repolarization at the soma but not the dendrites of hippocampal CA1 pyramidal neurons. J. Neurosci. 19: 5205-5212, 1999.

PumaIn, R. Calcium ions. In: The Neuronal Microenvironment, edited by A. Boulton, G. Baker, and W. Walz. Clifton, NJ: Humana, 1988, p. 589-641.

Pumain, R. AND HeinemanN, U. Stimulus- and amino acid-induced calcium and potassium changes in rat neocortex. J. Neurophysiol. 53: 1-16, 1985.

QIAN, J., Colmers, W. F., AND SAGGAU, P. Inhibition of synaptic transmission by neuropeptide $\mathrm{Y}$ in rat hippocampal area $\mathrm{CA} 1$ : modulation of presynaptic calcium entry. J. Neurosci. 17: 8169-8177, 1997.

Rose, S. Cell-adhesion molecules, glucocorticoids and long-term memory formation. Trends Neurosci. 18: 502-506, 1998.

SCHMOLKE, C. Morphological organization of the neuropil in laminae II-V of rabbit visual cortex. Anat. Embryol. 176: 203-212, 1987.

SMITH, S. J. Do astrocytes process neural information? In: Neuronal-Astrocytic Interactions. Implications for Normal and Pathological CNS Function, edited by A.C.H. Yu, L. Hertz, M. D. Norenberg, E. Sykova, and S. G. Waxman. New York: Elsevier, 1992, p. 119-136.

Smith, S. J. And Augustine, G. J. Calcium ions, active zones and neurotransmitter release. Trends Neurosci. 11: 458-464, 1988.

StAnLEy, E. F. Presynaptic calcium channels and the depletion of synaptic cleft calcium ions. J. Neurophysiol. 83: 477-482, 2000.

Stanton, P. And HeInemann, U. Norepinephrine enhances stimulus-evoked calcium and potassium concentration changes in dendate granule cell layer. Neurosci. Lett. 67: 233-238, 1986.

StUART, G. AND SaKmann, B. Active propagation of somatic action potentials into neocortical pyramidal cells dendrite. Nature 367: 69-72, 1994.

Svoboda, K., DenK, W., Kleinfeld, D., and Tank, D. In vivo dendritic calcium dynamics in neocortical pyramidal neurons. Nature 385: 161-165, 1997.

SYкоva, E. The extracellular space in the CNS: its regulation, volume and geometry in normal and pathological neuronal function. Neuroscientist 3: $28-41,1997$.

TANG, L., Hung, C. P., AND Schuman, E. M. A role for the cadherin family of cell adhesion molecules in hippocampal long-term potentiation. Neuron 20: 1165-1175, 1998.

Uchida, N., Honjo, Y., Johnson, K. R., Wheelock, M. J., and TAKeichi, M. The catenin/cadherin adhesion system is localized in synaptic junctions bordering transmitter release zones. J. Cell Biol. 135: 767-779, 1996.

Uemura, T. The cadherin superfamily at the synapse: more members, more missions. Cell 93: 1095-1098, 1998.

Vassilev, P. M., Mitchel, J., Vassilev, M., Kanazirska, M., and Brown, E. M. Assessment of frequency-dependent alterations in the level of extracellular Ca2+ in the synaptic cleft. Biophys. J. 72: 2103-2116, 1997.

XIONG, Z.-G., Lu, W.-Y., AND MacDonald, J. F. Extracellular calcium sensed by a novel cation channel in hippocampal neurons. Proc. Natl. Acad. Sci. USA 94: 7012-7017, 1997.

Yamagata, M., HeRmann, J. P., and SANES, J. R. Laminin-specific expression of adhesion molecules in developing chick optic tectum. J. Neurosci. 15: 4556-4571, 1995. 